\title{
Excited state mechanisms in crystalline carbazole: the role of aggregation and isomeric defects
}

\author{
Federico J. Hernández ${ }^{1}$ and Rachel Crespo-Otero*1 \\ ${ }^{1}$ Department of Chemistry, School of Biological and Chemical Sciences, Queen \\ Mary University of London, London, UK. Email: r.crespo-otero@qmul.ac.uk
}

\begin{abstract}
The molecule of Carbazole $(\mathrm{Cz})$ is commonly used as a building block in organic materials for optoelectronic applications, acting as light-absorbing, electron donor and emitting moiety. Crystals from $\mathrm{Cz}$ and derivatives display ultralong phosphorescence at room temperature. However, different groups have reported inconsistent quantum efficiencies for the same compounds. In a recent experimental study by Liu et al. (Nature Materials 2021, 20, 175-180), the ultralong phosphoresce properties of $\mathrm{Cz}$ have been associated with the presence of small fractions of isomeric impurities from commercially available $\mathrm{Cz}$. In this paper, we use state-of-the-art computational approaches to investigate light-induced processes in crystalline and doped $\mathrm{Cz}$. We revisit the role of aggregation and isomeric impurities on the excited state pathways and analyse the mechanisms for exciton, Dexter energy transfer and electron transport based on Marcus and Marcus-Levich-Jortner theories. Our excited state mechanisms provide a plausible interpretation for the experimental results and support the formation of charge-separated states at the defect/Cz molecular interface. These results contribute to a better understanding of the factors enhancing the excited state lifetimes in organic materials and the role of doping with organic molecules.
\end{abstract}

\section{INTRODUCTION}

The field of organic optoelectronics has experienced significant growth in the last decades. The development of novel organic luminophores, commonly, aggregated structures of $\pi$-conjugated materials in the solid phase, has boosted the design of new materials for technologies such as photovoltaics, solid-state lasers, phototherapy, molecular sensing, optical imaging, spintronics, amongst others [1-6]. Since some of these applications benefit from long-lived excited states, achieving high populations of triplet states presents significant advantages.

Metal-free organic chromophores have low spin-orbit couplings (SOCs) limiting the efficiency of intersystem crossing (ISC). However, different strategies can be implemented to promote ISC including designing donoracceptor systems with small singlet-triplet gaps and molecules with electron transitions that involve a change in the character of the states such as $n-\pi^{*}$ transitions (El-Sayed rule) [7]. Other pathways include heavy-atom and halogen substitution as well as crystal engineering [8-10].

The discovery of several pure organic roomtemperature phosphorescence (RTP) materials, displaying excited state lifetimes of over $100 \mathrm{~ms}$ in the crystalline phase, has attracted significant attention due to their promising applications [11-17]. Despite the recent experimental and theoretical efforts in this direction, the mechanisms behind ultralong organic phosphorescence (UOP), are yet to be fully understood.

Because of its extensive $\pi$-conjugation and easy derivatisation, $\mathrm{Cz}$ is a common building block in molecular materials for optoelectronic applications used as a light-absorbing, electron donor and emitting moiety

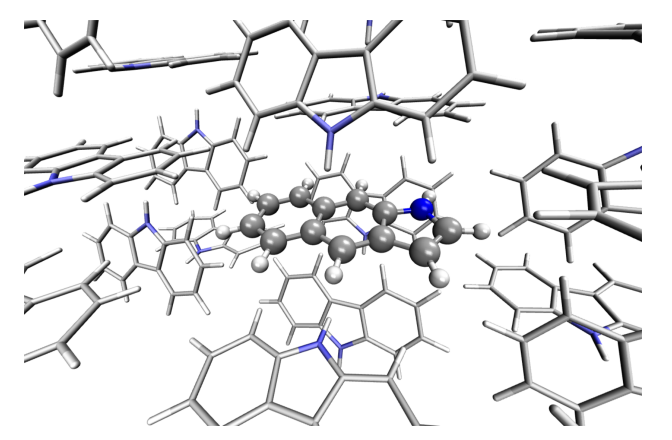

FIG. 1. Isomeric defect of Bd (1H-benzo[f]indole) highlighted with ball and stick representation.

$[1,8,18]$. Molecular crystals from pure $\mathrm{Cz}$ and its derivatives display UOP at room temperature [8, 19]. However, there are inconsistencies in the values of the lifetimes and quantum yields reported by different groups [20]. In a recent study, Liu et al. explored the role of isomeric defects in the UOP mechanism of $\mathrm{Cz}$. The authors found that the small fractions of $1 \mathrm{H}$-benzo[f]indole (Bd, Fig. 1) found in commercial $\mathrm{Cz}$ are responsible for the ultralong lifetimes of the excited states. The isomeric defects serve as microplanar heterojunctions facilitating the formation of chargeseparated states [20].

In organic semiconductors, defects enable the formation of localised carrier states acting as traps for electrons and holes [21]. The controlled use of defects to tune excited state lifetimes represents a promising opportunity in the field of functional electronic materials. To better understand the mechanism of formation of long-lived triplets in $\mathrm{Cz}$ crystals, we investigate the processes activated by light and the role of isomeric defects. We start by examining the mechanism following a localised excitation on the embedded monomers. We 
analyse the exciton states and transport and evaluate the role of $\mathrm{H}$-dimerisation on deactivation processes. We then investigate the formation of charge-transfer states in pure and doped crystalline $\mathrm{Cz}$ and the effect of isomeric defects of $\mathrm{Bd}$ on the excited state pathways. Our simulations help understand light-activated processes in crystalline $\mathrm{Cz}$ with implications in the design of new electronic materials for a broad range of applications.

\section{COMPUTATIONAL METHODS}

The crystal structure of $\mathrm{Cz}$, obtained from the CCDC (CCDC number 1525166), was optimised with periodic DFT using Quantum Espresso [22]. These calculations were done using the PBE-D2 functional with a plane wave cut-off of $40 \mathrm{Ry}$ and ensuring Monkhorst-Pack $k$ point convergence $(2 \times 1 \times 2)$.

To simulate the excited states in the molecular crystal, we created cluster models of $\mathrm{Cz}$ and applied the QM-QM' ONIOM schemes implemented in fromage $[23,24]$. The $\mathrm{QM}$ regions included either one or two molecules of $\mathrm{Cz}$ simulated at (TD-)B3LYP/6$311++\mathrm{G}(\mathrm{d}, \mathrm{p})$ level of theory using Gaussian 16 [25]. We tested different functionals including long-range corrected with optimal tuning. The B3LYP functional showed the best agreement with the experiments (section S1, Supporting Information). The cluster models considered 16 molecules for the case of one molecule in the QM region and 27 or 28 molecules for the QM regions including dimers. The QM' region was described using the density functional tight-binding formalism as implemented in the DFTB+ package [26].

The minima for the $\mathrm{S}_{0}, \mathrm{~S}_{1}, \mathrm{~S}_{2}$ and $\mathrm{T}_{1}$ states were optimised using the ONIOM Embedded Cluster model (OEC). The geometries of the $T_{3} / T_{2}$ and $S_{2} / S_{1}$ crossings were optimised with a penalty function with the condition of a zero energy gap [27]. The effect of the longrange electrostatic interactions was addressed using the ONIOM Ewald Embedded Cluster model (OEEC) [23]. For the electrostatic embedding at the higher level of theory, we use RESP charges obtained at the same level of theory. For the embedding of the lower level of theory, the charges were obtained with PBE/6-31G(d). Because long-range Coulomb interactions (model OEEC) do not have a significant effect on the energies (see section S3), we focus our discussion on the results obtained using the OEC model.

The probability of a radiative transition $k_{i f}$ (and the corresponding radiative lifetime from the $i$ state $\tau_{i}$ ) between an initial state $\left|\Psi_{i}\right\rangle$ and a final state $\left|\Psi_{f}\right\rangle$ can be calculated using the Fermi's Golden Rule [28]:

$$
k_{i f}(\omega)=\frac{4 \omega^{3} n^{2}}{3 \hbar c^{3}}\left|\left\langle\Psi_{i}|\hat{\mu}| \Psi_{f}\right\rangle\right|^{2} \delta\left(E_{i}-E_{f} \pm \hbar \omega\right),
$$

where $\omega$ is the frequency of the photon, $n$ is the refractive index of the medium, $\hbar$ is the Planck constant divided by $2 \pi, c$ is the speed of light in vacuum, $\mu$ is the electric transition dipole moment operator, and $E_{j}$ is the energy of the state $j$. We considered a refractive index of $n=1.781$ for the crystal [29]. For fluorescence, $\vec{\mu}_{i \rightarrow f}=\left\langle S_{n}|\hat{\mu}| S_{0}\right\rangle$, where $S_{n}$ is the emitting state, and in most cases $n=1$ (Kasha's rule).

In the case of phosphorescence, $\left\langle T_{m}|\hat{\mu}| S_{0}\right\rangle$ can be approximated using the first-order peturbation theory to include the spin-orbit coupling operator $\hat{H}_{S O C}$ allowing intensity borrowing from spin-allowed transitions [30]. This approximation converges slowly with the number of excited states and significant errors can be obtained even when hundred of excited states are included [31, 32]. In this work, we use instead the multiconfigurational quadratic response (MCQR) method, which provides reliable values for $\mu_{T_{m} \rightarrow S_{0}}$ [32]. These calculations were performed at the TD-B3LYP/6-311++G(d,p) level of theory using the Dalton software [33, 34].

Herein, we estimate the rate constants for nonradiative electron transfer (ET) processes as intersystem crossing (ISC), and transport processes like exciton and charge transfer using the semiclassical Marcus theory as:

$$
k_{E T}=\frac{2 \pi}{\hbar}\left|H_{a b}\right|^{2} \frac{1}{\sqrt{4 \pi \lambda k_{B} T}} \exp \left(-\frac{\left(\Delta G^{\circ}+\lambda\right)^{2}}{4 \lambda k_{B} T}\right),
$$

where $\mathrm{T}$ is the absolute temperature, $k_{B}$ is the Boltzmann constant, $\lambda$ is the reorganisation energy induced by the population transfer and $\Delta G^{\circ}$ stands for the variation of the adiabatic Gibbs free energy during the process. $H_{a b}$ is the electronic coupling term between the diabatic states involved in the ET process.

For ISC, $H_{a b}=\left\langle S_{n}\left|\boldsymbol{H}_{\boldsymbol{S O C}}\right| T_{m}\right\rangle$, where $\mathrm{S}_{n}$ and $\mathrm{T}_{m}$ are the singlet and triplet states involved in the $\mathrm{S}_{n} \rightarrow \mathrm{T}_{m}$ nonradiative transition. The reorganisation energy was approximated as $\lambda \approx E_{T m}\left(\mathbf{R}_{\min S_{n}}\right)-E_{T m}\left(\mathbf{R}_{\min T_{m}}\right)$, as it has been done in previous works [35]. The spin-orbit couplings (SOCs) were evaluated at the TDB3LYP $/ 6-311++G(d, p)$ level of theory adopting the Breit-Pauli spin-orbit Hamiltonian with effective charge approximation as implemented in the PySOC package [36].

The ET rate coefficients were also calculated using the Marcus-Levich-Jortner (MLJ) equation as implemented in references [37] and [38]:

$$
k_{E T}=\frac{2 \pi}{\hbar}\left|H_{a b}\right|^{2} F_{k},
$$

where $F_{k}$ is the final vibrational state density at the point of the initial state energy level weighted by Franck-Condon factors. The vibrational modes with frequencies well above $210 \mathrm{~cm}^{-1}\left(k T_{300 K}\right)$ and the highest reorganisation energies were considered as quantum modes $q_{j}$ as explained in the section S2 of the Supporting Information. The Huang-Rhys factors and reorganisation energies were calculated using the 
DUSHIN code [39].

For the calculation of the exciton hopping rates of the singlet and triplet states, the coupling terms $\left(H_{a b}=J_{i j}^{S}\right.$ and $H_{a b}=J_{i j}^{T}$ ) were evaluated using the half energy splitting method. The coupling terms used to evaluate the energy transfer rates of triplets $\left(H_{a b}^{T}\right)$ or charge hopping rates $\left(H_{a b}^{C T}\right)$ were computed using constrained DFT with configuration interactions (CDFT-CI) as implemented in Q-Chem [40]. The active space considered for the CDFT-CI calculations of the couplings between the triplets was $\left\{\left|S_{0} S_{0}\right\rangle,\left|S_{0} T_{1}\right\rangle,\left|T_{1} S_{0}\right\rangle\right\}$. For the calculation of the charge transfer between negatively charged $\left(C z^{-}\right)$and neutral molecules, the active space compromised the following states $\left\{\left|S_{0} C z^{-}\right\rangle,\left|C z^{-} S_{0}\right\rangle\right\}$. To analyse the performance of these methods, additional single point calculations were performed using the $\mathrm{ADC}(2) / \mathrm{TZVP}$ level of theory with point charge embedding as implemented in Turbomole [41].

The fluorescence time constant $\left(\tau_{f}\right)$ and quantum yield $\left(\Phi_{f}\right)$ were obtained using the following equations:

$$
\begin{gathered}
\tau_{f}=\frac{1}{k_{f}+k_{I C}+k_{I S C}} \\
\Phi_{f}=\frac{k_{f}}{k_{f}+k_{I C}+k_{I S C}}=k_{f} \tau_{f}
\end{gathered}
$$

where $k_{f}$ is the fluorescence rate coefficient, $k_{I C}$ stands for the internal conversion rate coefficient from the excited singlet state $\mathrm{S}_{n}$ to the ground state $\mathrm{S}_{0}, k_{I S C}$ is the global intersystem crossing rate coefficient from $\mathrm{S}_{n}$ to all the possible triplet states involved. Based on the experimental observations in gas phase and solution, the internal conversion from $\mathrm{S}_{1}$ to $\mathrm{S}_{0}$ was neglected $\left(k_{I C}=0\right)$ $[42,43]$. with:

The phosphorescence time constant $\left(\tau_{p}\right)$ was obtained

$$
\tau_{p}=\frac{1}{k_{p}+k_{I S C}^{\prime}+k_{I C}^{\prime}}
$$

where $k_{p}$ is the phosphorescence rate coefficient, $k_{I C}^{\prime}$ is the internal conversion rate coefficient from the excited triplet state $\mathrm{T}_{m}$ to another excited triplet state of lower energy $\mathrm{T}_{l}, k_{I S C}^{\prime}$ is the intersystem crossing rate coefficient for $\mathrm{T}_{m} \rightarrow \mathrm{S}_{0}$. We assumed $k_{I C}^{\prime}=0$ because $\tau_{p}$ was computed from the $\mathrm{T}_{1}$ state.

We have also estimated the diffusion constant in the singlet or triplet state as

$$
D=\frac{k_{i j} R_{i j}^{2}}{z},
$$

where $k_{i j}$ is the exciton hopping rate coefficient between the donor $i$ and the acceptor $j, R_{i j}$ is the distance between the centre of masses of $i$ and $j$ and $z$ is a parameter equal to 2,4 or 6 for one, two and three-dimensional diffusion, respectively. Equation 7 was derived considering nearest neighbour random walk hopping in an isotropic medium for a cubic cell [44]. Herein, we use it to provide a qualitative comparison between transport in singlet and triplet states. The diffusion length was calculated as

$$
L_{D}=\sqrt{D \tau}
$$

where $\tau$ is the fluorescence or phosphorescence lifetime.

\section{RESULTS AND DISCUSSION}

Given the weak nature of intermolecular interactions in molecular organic crystals, localised excitations and incoherent exciton transfer are common mechanisms at room temperature [45]. We have considered the formation of excited species localised on monomers and dimers in the $\mathrm{Cz}$ crystal as the initial step in the excited state mechanism. In the next section, we explore the excited state mechanism following the excitation of a single monomer in the crystal environment.

\section{A. The molecule centred mechanism}

To understand the excited processes, we explored the linear interpolated pathway between the ground state and the excited state minima and also evaluated the SOCs between the triplets and $\mathrm{S}_{1}$ (Fig. 2). There is a good agreement between the calculated energies at TD-B3LYP $/ 6-311++\mathrm{G}(\mathrm{d}, \mathrm{p})$ level of theory and the experimental data (Table S3) [18-20,46]. A value of $3.73 \mathrm{eV}$ is obtained for the $\mathrm{S}_{0} \rightarrow \mathrm{S}_{1}$ absorption energy of a cluster including 6 molecules of $\mathrm{Cz}$ in contrast with the experimental value of $3.62 \mathrm{eV}$.

The experimental oscillator strength for the $\mathrm{S}_{0} \rightarrow \mathrm{S}_{2}$ transition in the gas phase is an order of magnitude greater than that for $\mathrm{S}_{0} \rightarrow \mathrm{S}_{1}$ [42]. A similar trend is observed in solid state $[18,19]$. Depending on the initial energy, either the $S_{1}$ or the brightest $S_{2}$ state can be populated. Provided initial excitation to $S_{1}$, the system will follow vibrational relaxation in the picosecond scale to the $\mathrm{S}_{1 \min }$ from which fluorescence can compete with non-radiative mechanisms and proccesses like exciton transfer (see Section IIIB). Experiments in the gas phase using jet-cooled molecular beams do not show phosphorescence or nonradiative decay to $\mathrm{S}_{0}\left(\phi_{f} \approx 1\right)$ [42]. However, ISC is the main non-radiative pathway from $S_{1}$ in the condensed phases $[18,19,43,46]$.

Fluorescence lifetime $\left(\tau_{f}\right)$ of $\mathrm{Cz}$ in solution under inert atmosphere or dispersed in a solid matrix is around 14$15 \mathrm{~ns}$, with a quantum yield of $\Phi_{f}=0.4-0.6$. When $\mathrm{Cz}$ is dissolved in organic media under air saturated 
atmosphere, $\tau_{f}=7.7-8.8 \mathrm{~ns}$ with $\Phi_{f}=0.28-0.48$ [43]. In the crystalline phase, $\tau_{f}=8.2 \mathrm{~ns}$ at $300 \mathrm{~K}$ with a $\Phi_{f}=$ 0.78 [18]. For the pure crystal, we obtained fluorescence lifetimes of 11.1 and 7.9 ns (Equation 4) and fluorescence quantum yields of 0.76 and 0.55 considering Marcus of MLJ model, respectively. These values are in excellent agreement with the experimental ones (Table I), which indicates that ISC is the most important competing pathway.

TABLE I. Fluorescence lifetime $\left(\tau_{f}\right)$ and quantum yield $\left(\Phi_{f}\right)$ computed using Equations 4 and 5 with $k_{I C}=0$. Phosphorescence lifetime $\left(\tau_{p}\right)$. $k_{I S C}$ was calculated using the Equation 2. The values in brackets were obtained computing $k_{I S C}$ with Equation 3.

\begin{tabular}{lcc}
\hline \hline & Predicted & Experimental \\
\hline$\tau_{f}$ & $11.1(7.9) \mathrm{ns}$ & $8.2-11.5 \mathrm{~ns}[18,19]$ \\
$\Phi_{f}$ & $0.76(0.55)$ & $0.78[18]$ \\
$\tau_{p}$ & $7.2(7.2) \mathrm{s}$ & $1.11 \mathrm{~s}^{\dagger}[18]$ \\
$k_{I S C}$ & $2.1410^{7}\left(5.7510^{7}\right) \mathrm{s}^{-1}$ & $3.8710^{7} \mathrm{~s}^{-1 \dagger \dagger}[43]$ \\
\hline
\end{tabular}

${ }^{\dagger}$ Measured in solid matrix at $4 \mathrm{~K}$. The value at $300 \mathrm{~K}$ is $0.852 \mathrm{~s}$

${ }^{\dagger \dagger}$ Measured in solid matrix at $77 \mathrm{~K}$

At the $\mathrm{S}_{1}$ minimum, the SOCs are smaller than $1 \mathrm{~cm}^{-1}$, which is not surprising in the light of the El Sayed rule given the $\pi-\pi^{*}$ character of all states involved. The SOC values between $S_{1}$ and $T_{n=1,2,3}$ remains almost unaltered between the $\mathrm{FC}$ and $\mathrm{S}_{1 \text { min }}$ geometries (Fig. 2b). Due to the similar energies of $\mathrm{S}_{1}$ and $\mathrm{T}_{3}\left(\Delta E_{S_{1}-T_{3}}=0.07\right.$ $\mathrm{eV}$ ), direct intersystem crossing is likely to occur (Fig. 2a). While the SOC values with $\mathrm{T}_{1}$ are almost 4 times greater than those with $\mathrm{T}_{3}$, a large energy gap $(0.77 \mathrm{eV})$ prevents the ISC to occur to $T_{1}$. The rate for the $S_{1} \rightarrow T_{3}$ transition calculated with Marcus and MLJ equations are $2.1410^{7} \mathrm{~s}^{-1}$ and $5.7510^{7} \mathrm{~s}^{-1}$ respectively. These values are in line with the ISC rate of $3.8710^{7} \mathrm{~s}^{-1}$ obtained in a solid matrix of iso-propanol-ethyl ether and rates obtained for $\mathrm{Cz}$ derivatives in the solid state (Table I) $[18,43]$.

The existence of a region for the crossing between both surfaces $\left(T_{3} / T_{2}\right)$ promotes internal conversion from $T_{3}$ to $\mathrm{T}_{2}$. The optimised geometry of the crossing shows a slight out-of-plane distortion from one of the aromatic rings (Figure S6). After the crossing, the population can split into two branches (right or left, Figure 2 ), resulting in either phosphorescence or nonradiative decay. The band associated with phosphorescence from $\mathrm{T}_{1 \text { min }}$ appears at $2.25 \mathrm{eV}$ [18] (calculated value $2.57 \mathrm{eV}$ ).

Crystalline $\mathrm{Cz}$ also shows a phosphorescence sideband at $2.95 \mathrm{eV}$, in very good agreement with the predicted emission from $\mathrm{T}_{2}$ found at $2.98 \mathrm{eV}$ [19]. This band becomes more intense when the crystal is electronically excited at energies over the $\mathrm{S}_{1}$ excitation energy (3.62 eV) [19]. Initial excitation to $\mathrm{S}_{2}$ could be followed either by $\mathrm{IC}$ to $\mathrm{S}_{1}$, the most likely pathway, or ISC to $\mathrm{T}_{4}$. The linear interpolated pathway considering relaxation from $\mathrm{S}_{2}$ is shown in Figure S5. An appreciable vibronic coupling has been observed between $\mathrm{S}_{2}$ and $\mathrm{S}_{1}$ states

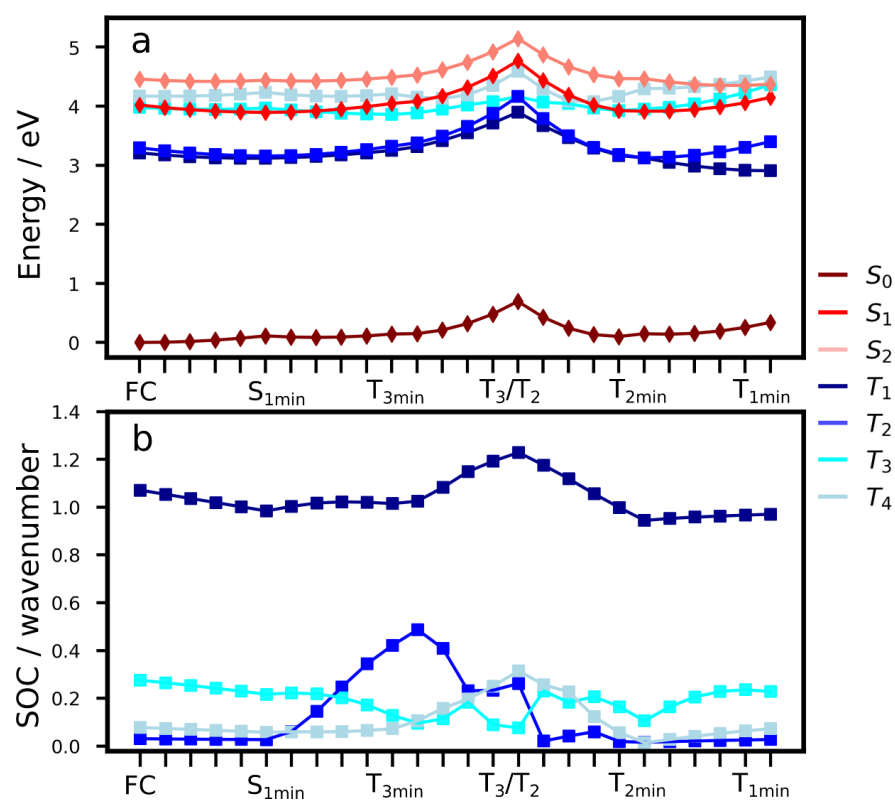

FIG. 2. (a) Linear interpolated Cartesian coordinates pathway (LICC) from the Franck-Condon (FC) geometry, to $\mathrm{S}_{1}$, to the excited state minima (OEC model, TD-B3LYP/6$311++\mathrm{G}(\mathrm{d}, \mathrm{p})$ ) (b) Spin-orbit couplings values along the LICC pathway.

in gas-phase [42], and solid state as well [47]. We have also located an accessible $\mathrm{S}_{2} / \mathrm{S}_{1}$ crossing, which can help facilitate IC (Figures S5 and S6). Once in $\mathrm{S}_{1}$, the system can explore the relaxation pathways described before. Our estimated value of the ISC rate for the $\mathrm{S}_{2} \rightarrow \mathrm{T}_{4}$ transition is $k_{I S C}^{S_{2}}=7.310^{7} \mathrm{~s}^{-1}$ which is just 3.3 times greater than $k_{I S C}^{S_{1}}$. At the $\mathrm{S}_{2 \min }$ structure, $\mathrm{S}_{2}$ and $\mathrm{T}_{4}$ have very similar energies $\left(\Delta E_{S_{2}-T_{4}}=-0.008 \mathrm{eV}\right)$.

Once the system is in the lowest energy triplet manifold, provided the excess of vibrational energy, the molecule will be able to explore the left brand for a longer time allowing for phosphorescence emission at higher energies $(2.98 \mathrm{eV})$, which explains the sideband in the phosphorescence spectrum. The energies of $\mathrm{T}_{1}$ and $\mathrm{T}_{2}$ are almost degenerate and very close to the energy of the $\mathrm{T}_{2}$ minimum. Non-Kasha phosphorescence has been also observed for analogue systems based on dibenzothiophene, where a sideband shifted to the blue from the $\mathrm{T}_{1}$ emission band was unambiguously assigned to emission from $\mathrm{T}_{2}$ [48].

\section{B. Exciton processes}

\section{Dimers: Is H-aggregation relevant?}

$\mathrm{H}$-aggregation has been hypothesised as the reason for the stabilisation of long-lived triplet states in crystalline $\mathrm{Cz}$ and UOP materials in general $[19,49]$. The $\mathrm{Cz}$ molecule crystalises in a herringbone packing motif (Fig. 
3) [46]. Herein, we investigate how the aggregation patterns affect the excited state mechanism of crystalline $\mathrm{Cz}$.
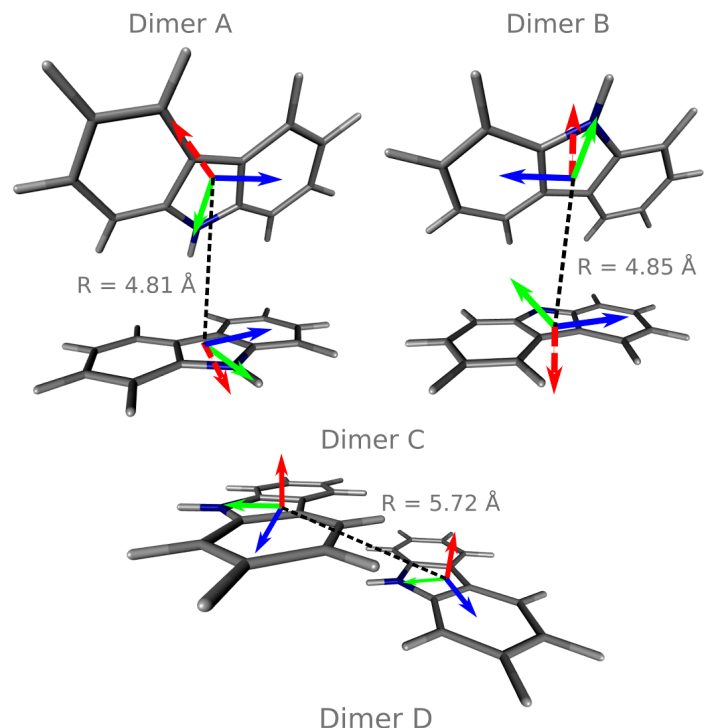

Dimer D
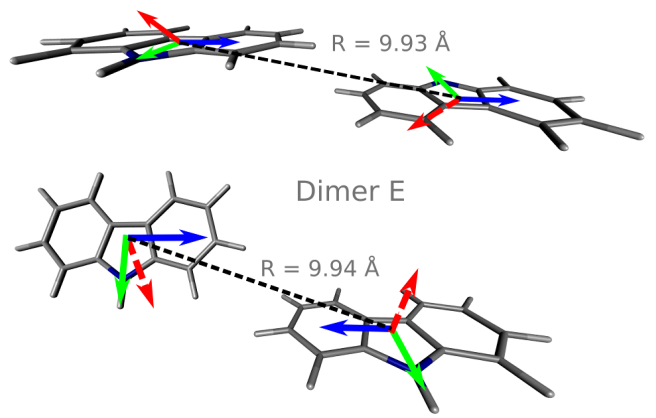

Dimer F

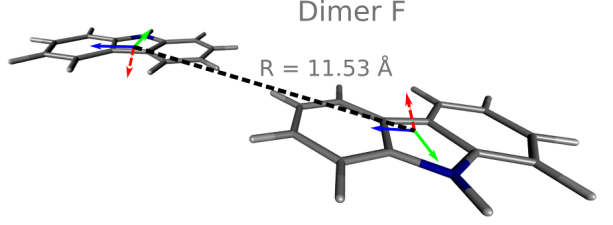

FIG. 3. Molecular dimers in the $\mathrm{Cz}$ crystal optimised at the TD-B3LYP/6-311++G(d,p) level within the OEC model. Distances between the centre of masses $(R)$ are also shown. The green, blue and red arrows represent the transition dipole moments of the $\mathrm{S}_{0} \rightarrow \mathrm{S}_{1}, \mathrm{~S}_{0} \rightarrow \mathrm{S}_{2}$ and the $\mathrm{T}_{1}$ $\rightarrow \mathrm{S}_{0}$ transitions, respectively. The arrow lengths are not proportional to the transition dipole magnitudes.

We have identified six different dimers (A-F, Fig. 3). The dimers $\mathrm{A}, \mathrm{B}$, and $\mathrm{C}$ have been previously reported [19, 20]. Dimers A, B, and E show edge-to-face arrangements commonly found in herringbone crystals. While Dimers C and D are slipped $\pi$-stack face-to-face structures. Dimer $\mathrm{F}$ is planar displaying a close $\mathrm{H}$..H contact at $3.19 \AA$. Table II displays the splitting of the first excited state into the excitonic states $S_{1}$ and $S_{2}$. A very small red-shift of the absorption energy is predicted. For the singlet states, $\mathrm{S}_{1}$ and $\mathrm{S}_{2}$ are excitonic states. The largest exciton couplings are obtained for dimers B (4
TABLE II. Photophysical properties of the six different dimers motifs present in the crystal packing of carbazole. $E_{S_{1}}$ and $E_{S_{2}}$ are the absorption energies of the lower and upper states produced by exciton splitting. $f_{S_{1}}$ and $f_{S_{2}}$ are the corresponding oscillator strengths. The aggregates were classified spectroscopically according to their oscillator strengths. The angle $\theta$ was evaluated at the respective $\mathrm{S}_{0}$ equilibrium geometry of each dimer

\begin{tabular}{lccccccc}
\hline \hline Dimer & $E_{S_{1}}(\mathrm{eV})$ & $f_{S_{1}}$ & $E_{S_{2}}(\mathrm{eV})$ & $f_{S_{2}}$ & Aggregate & \multicolumn{2}{c}{$\theta$} \\
& & & & & Type & Mon 1 & Mon 2 \\
\hline $\mathrm{A}$ & 4.010 & 0.032 & 4.011 & 0.016 & $\mathrm{~J}$ & $28.9^{\circ}$ & $79.7^{\circ}$ \\
$\mathrm{B}$ & 4.001 & 0.032 & 4.009 & 0.012 & $\mathrm{~J}$ & $26.5^{\circ}$ & $77.1^{\circ}$ \\
$\mathrm{C}$ & 3.972 & 0.077 & 4.019 & 0.001 & $\mathrm{~J}$ & $27.8^{\circ}$ & $26.7^{\circ}$ \\
$\mathrm{D}$ & 4.021 & 0.000 & 4.023 & 0.058 & $\mathrm{H}$ & $83.0^{\circ}$ & $83.1^{\circ}$ \\
$\mathrm{E}$ & 4.018 & 0.018 & 4.020 & 0.044 & $\mathrm{H}$ & $72.0^{\circ}$ & $77.6^{\circ}$ \\
$\mathrm{F}$ & 4.019 & 0.000 & 4.020 & 0.063 & $\mathrm{H}$ & $57.1^{\circ}$ & $57.1^{\circ}$ \\
\hline \hline
\end{tabular}

$10^{-3}$ ) and $\mathrm{C}\left(2.310^{-2}\right)$ (Tables II and III).

Using the spectroscopic classification based on the oscillator strength values, the dimers can be classified as H- (D, E and F) or J-aggregates (A, B and C). The Haggregates are obtained when $f_{S_{2}}>f_{S_{1}}$ and J-aggregates when $f_{S_{1}}>f_{S_{2}}$. Previous studies on the effect of $\mathrm{H}$ aggregation in UOP organic materials have considered the classical Kasha's exciton model [19, 49], which is based on the analysis of Coulombic interactions between two chromophores:

$$
J_{\text {Coul }}=\frac{\boldsymbol{\mu}_{\mathbf{1}} \cdot \boldsymbol{\mu}_{\mathbf{2}}-3\left(\boldsymbol{\mu}_{\mathbf{1}} \cdot \hat{R}\right)\left(\boldsymbol{\mu}_{\mathbf{2}} \cdot \hat{R}\right)}{4 \pi \epsilon R^{3}},
$$

where $\boldsymbol{\mu}_{\boldsymbol{i}}$ is the transition dipole moment (TDM) vector of the molecule " $i$ ", $\vec{R}=R \hat{R}$ is the intermolecular displacement vector connecting the centres of mass of the monomers, and $\epsilon$ is the optical dielectric constant of the medium. For co-planar TDM vectors, the angle $\theta$ is defined as the angle between $\vec{R}$ and the TDM vector of any of the chromophores and the $J_{\text {Coul }}$ is given by

$$
J_{\text {Coul }}=\frac{\mu^{2}\left(1-3 \cos ^{2} \theta\right)}{4 \pi \epsilon R^{3}} .
$$

From Equation 10 emerges the $\mathrm{H} / \mathrm{J}$ classification based on the magic angle $\theta=54.7^{\circ}$, i.e., J-aggregates are formed if $0^{\circ}<\theta<54.7^{\circ}$ and $\mathrm{H}$-aggregates if $54.7^{\circ}<$ $\theta<90.0^{\circ}$, which is strictly valid for systems with coplanar TDMs [50]. The transition dipole moments for dimers $\mathrm{A}$ and $\mathrm{B}$ are oblique and define different planes (Fig. 3). Because of this, the spectroscopic classification of the dimers $\mathrm{A}$ and $\mathrm{B}$ does not agree with the obtained with the Kasha's angle (Table II). For F, the TDMs are co-planar either, however in this case the angle $\theta$ is close to the magic angle of 54.7.

The exchange interactions not considered in Kasha's model could be relevant for these dimers with intermolecular distances around $3 \AA$ (Figure S8). Close 
packing arrangements are susceptible to experience a significant wave function overlap and therefore, shortrange interactions as exchange or superexchange. The magnitude of the short-range coupling can be comparable or even exceed the Coulomb coupling. These interactions can promote a different exciton behaviour than normally associated with Kasha's H/J-aggregates [51], thus the conventional geometric classification is incomplete at best.

In the case of the triplet states for the dimers A, B, C and $\mathrm{E}$, the lowest energy states are localised and we could not find exciton states. For the dimers D and F, where the states $\mathrm{T}_{1}-\mathrm{T}_{6}$ are excitonic, the values of $J$ are smaller than $2.510^{-4} \mathrm{eV}$ except for the $\mathrm{T}_{5}-\mathrm{T}_{6}$ splitting with $J_{T_{5} T_{6}}=1.010^{-3} \mathrm{eV}$. We optimised the geometries of the $\mathrm{T}_{1}$ and $\mathrm{S}_{1}$ states of all dimers in the crystal environment. In line with the experimental observations, the predicted phosphorescence from $T_{1}$ is an out-of-plane polarised transition (Fig. 3) [52]. Dimerisation has a minimal effect on the energy of the $\mathrm{T}_{1} \rightarrow \mathrm{S}_{0}$ transition and the corresponding phosphorescence lifetimes (see Table S7). The SOCs values between $S_{1}$ and triplets with lower or similar energies are not considerably increased with respect to the monomer (Tables S4 and S6). Hence, no increase in the ISC rate constant is expected via direct SOC due to the presence of dimers though the number of ISC channels could be greater.

For embedded dimers, the $\mathrm{T}_{1} \rightarrow \mathrm{S}_{0}$ transition densities are localised on single molecules. A significant degree of localisation is also found for a cluster of $\operatorname{six} \mathrm{Cz}$ molecules all of them treated quantum mechanically (Fig. $\mathrm{S} 10)$. Our calculations show $\mathrm{H} / \mathrm{J}$ - aggregation does not have a relevant effect on the energies or lifetimes of the triplet states of $\mathrm{Cz}$. In the next section, we analyse the implications of the localisation of the excitations for different transport regimes for singlets and triplets in pure crystalline $\mathrm{Cz}$.

\section{Exciton transport}

Exciton transfer competes with radiative and nonradiative molecule-centred processes in the molecular crystals. Exciton transport can proceed via coherent or incoherent hopping. In the latter case, it occurs mainly via Coulomb coupling which in its most simple form can be modelled by Equation 9, obtaining a $R^{-6}$ decay of the hopping rate [53]. Due to the lower concentration of triplets and their weak exciton couplings in $\mathrm{Cz}$, triplet diffusion is likely to proceed through a Dexter energy transfer (triplet-singlet) instead of a triplet-triplet diffusion process. Triplet diffusion is not theoretically limited to $100-200 \mathrm{~nm}$ as in singlet excitons because the SOCs, which determines the triplet lifetimes, do not govern the transport of triplets [54].

To evaluate the rates of singlet exciton transport and triplet energy transfer in $\mathrm{Cz}$ crystals, we have considered the exciton coupling rates between adjacent molecules using the Marcus and MLJ models (Equations 2 and 3). Table III shows the exciton hopping rates $k_{i j}$, diffusion coefficients $D$ and diffusion lengths $L_{D}$ for $\mathrm{S}_{1}$ and $\mathrm{T}_{1}$ at 298 K. $D$ and $L_{D}$ are obtained with Equations 7 and 8 , respectively, using the distances between monomers shown in Fig. 3 and the experimental radiative lifetimes (Table I). For both singlets and triplets, the hopping rate coefficients are systematically greater when the MLJ model is used. This shows the active role of vibrations in the exciton hopping process because the process is accompanied with a concomitant molecular distortion, i. e. the motion should have a polaronic character and thermally activated diffusion could be important.

TABLE III. Exciton couplings $\left(J_{i j}\right)$, exciton hopping rate coefficients $\left(k_{i j}\right)$, diffusion constants $(D)$ and diffusion lengths $\left(L_{D}\right)$ computed for the six dimers present in the crystal packing using Equations 2, 7 and 8, respectively, at the TD-B3LYP/6-311++G(d,p) level of theory. The values in brackets are obtained computing $k_{i j}$ with Equation 3.

\begin{tabular}{|c|c|c|c|c|}
\hline \multicolumn{5}{|c|}{ Singlets } \\
\hline Dimer & $J_{i j}^{S}(\mathrm{eV})$ & $k_{i j}^{S}\left(\mathrm{~s}^{-1}\right)$ & $D^{S}\left(\mathrm{~cm}^{2} \mathrm{~s}^{-1}\right)$ & $L_{D}^{S}(\mathrm{~nm})$ \\
\hline $\mathrm{A}$ & $1.010^{-3}$ & $7.810^{8}\left(4.510^{9}\right)$ & $9.010^{-7}\left(5.210^{-6}\right)$ & $1(2)$ \\
\hline B & $4.010^{-3}$ & $5.010^{10}\left(2.910^{11}\right)$ & $5.910^{-5}\left(3.410^{-4}\right)$ & $8(20)$ \\
\hline $\mathrm{C}$ & $2.310^{-2}$ & $1.710^{12}\left(9.510^{12}\right)$ & $2.710^{-3}\left(1.610^{-2}\right)$ & $56(134)$ \\
\hline $\mathrm{D}$ & $1.010^{-3}$ & $3.110^{9}\left(1.810^{10}\right)$ & $1.510^{-5}\left(8.910^{-5}\right)$ & $4(10)$ \\
\hline $\mathrm{E}$ & $1.010^{-3}$ & $3.110^{9}\left(1.810^{10}\right)$ & $1.510^{-5}\left(8.910^{-5}\right)$ & $4(10)$ \\
\hline $\mathrm{F}$ & $1.010^{-3}$ & $7.810^{8}\left(4.510^{9}\right)$ & $5.210^{-6}\left(\begin{array}{lll}3.0 & \left.10^{-5}\right)\end{array}\right.$ & $2(6)$ \\
\hline \multicolumn{5}{|c|}{ Triplets } \\
\hline Dimer & $H_{a b}^{T}(\mathrm{eV})$ & $k_{i j}^{T}\left(\mathrm{~s}^{-1}\right)$ & $\overline{D^{T}\left(\mathrm{~cm}^{2} \mathrm{~s}^{-1}\right)}$ & $\overline{L_{D}^{T}(\mathrm{~nm})}$ \\
\hline $\mathrm{A}$ & $\approx 0$ & $\approx 0$ & $\approx 0$ & $\approx 0$ \\
\hline B & $4.210^{-4}$ & $7.410^{6}\left(1.310^{8}\right)$ & $8.710^{-9}\left(1.610^{-7}\right)$ & $979(4179)$ \\
\hline $\mathrm{C}$ & $2.310^{-3}$ & $2.310^{8}\left(4.110^{9}\right)$ & $3.710^{-7}\left(6.810^{-6}\right)$ & $6390(27282)$ \\
\hline $\mathrm{D}$ & $1.210^{-4}$ & $6.010^{5}\left(1.110^{7}\right)$ & $2.910^{-9}\left(5.410^{-8}\right)$ & $569(1399)$ \\
\hline $\mathrm{E}$ & $\approx 0$ & $\approx 0$ & $\approx 0$ & $\approx 0$ \\
\hline $\mathrm{F}$ & $\approx 0$ & $\approx 0$ & $\approx 0$ & $\approx 0$ \\
\hline
\end{tabular}

The total reorganisation energy for exciton transfer in $\mathrm{S}_{1}$ is $\lambda=0.246 \mathrm{eV}$, which corresponds to an approximate barrier of $\lambda / 4=0.0615 \mathrm{eV}$ for the exciton hopping between adjacent molecules. This barrier is larger than the values of exciton couplings for all dimers. Thus, a significant localisation of the singlet excitation is expected and exciton transport should proceed through an incoherent hopping mechanism. In our calculations, six modes with frequencies 670, 1035, 1307, 1337, 1371 and $1661 \mathrm{~cm}^{-1}$ associated with in-plane bending and stretching vibrations of the $\mathrm{C}-\mathrm{C} / \mathrm{C}=\mathrm{C} / \mathrm{C}=\mathrm{N}$ bonds of the $\pi$ conjugated ring had the highest Huang-Rhys factors and were treated as quantum modes (see section S2 and Fig. S7). The largest value of $J$ is obtained for dimer $\mathrm{C}$ due to its $\pi$-stack structure. For dimer $\mathrm{C}$, the $k_{i j}$ is comparable to the obtained for the analogue slipped $\pi$-stacked dimer of anthracene, which also features a herringbone structure and incoherent exciton transport mechanism [38].

Fig. 4 shows possible exciton transfer paths through the dimers with the shortest intermolecular distances (A, $\mathrm{B}$ and $\mathrm{C}$ ). The predicted exciton rate coefficients (Table III) indicate that the only process likely to compete with vibrational relaxation to $\mathrm{S}_{1 \min }$ (taking $h \nu_{v i b} \approx 10^{3} \mathrm{~cm}^{-1}$ 
leads to $2 \pi / \nu_{v i b} \approx 210^{-13} \mathrm{~s}$ ) is exciton transport along dimer $\mathrm{C}$ preventing fluorescence or ISC to occur. Exciton hopping through dimer $\mathrm{B}$ involves a further transfer through the dimer A (Fig. 4 a), which has a very small exciton coupling and hence a small hopping rate coefficient. The 1-D diffusion coefficients and diffusion lengths obtained considering exciton transport in $\mathrm{S}_{1}$ for dimer $\mathrm{C}$ are $2.710^{-3} \mathrm{~cm}^{2} \mathrm{~s}^{-1}$ and $134 \mathrm{~nm}$, respectively, in qualitative agreement with the values experimentally and computationally reported for pure anthracene crystals [38]. Exciton transport is also anisotropic in anthracene, where the slipped $\pi$-stacked dimer similar to dimer $\mathrm{C}$ also shows the higher diffusion constant and length [38].

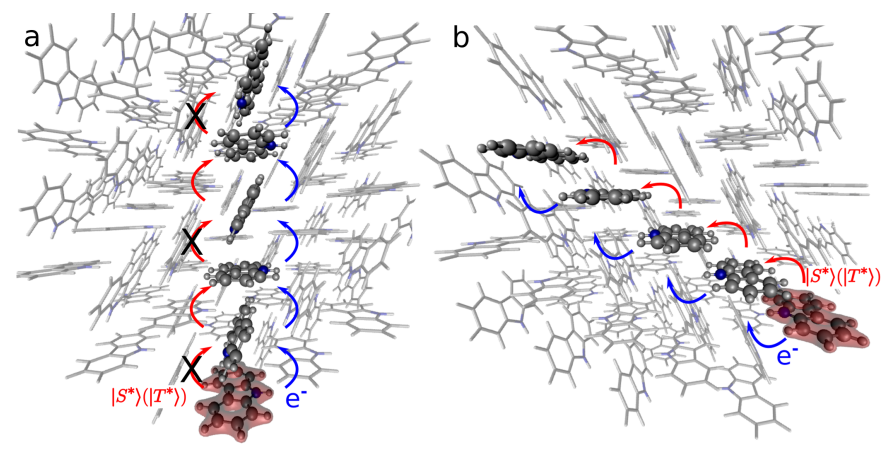

FIG. 4. Schematic representation of two possible exciton transport pathways involving dimers $\mathrm{A}$ and $\mathrm{B}(\mathrm{a})$, or dimer $\mathrm{C}$ (b). The highlighted molecule represents the initial position for electron ( $\mathrm{e}^{-}$, in Blue) or excitaton $\left(\left|S^{*}\right\rangle\right.$ or $\left.\left|T^{*}\right\rangle\right)$ transport (in red). The crosses indicate specific pathways that are less likely due to the small values of the couplings.

In the case of the $T_{1}$, the rates were calculated considering $\lambda=0.643 \mathrm{eV}$ and the normal mode with $\omega_{k}=$ $1661 \mathrm{~cm}^{-1}$ with the highest Huang-Rhys factor (Figure $\mathrm{S} 7$ ), which involves the in-plane stretching vibration of the $\mathrm{C}-\mathrm{C} / \mathrm{C}=\mathrm{C} / \mathrm{C}=\mathrm{N}$ bonds of the ring. Due to their lower couplings and the higher reorganisation energies, Dimers B, C, and D show hopping rates three orders of magnitude smaller in $\mathrm{T}_{1}$ than in $\mathrm{S}_{1}$ (see Table III). The small couplings in dimers $\mathrm{A}, \mathrm{E}$ and $\mathrm{F}$ are caused by a small overlap between the constituent monomers, preventing any energy transfer in these directions (see Fig. 4a).

Due to the spin-forbidden nature of phosphorescence, triplets exhibit lifetimes on the order of milliseconds for many of the pure organic materials with potential use in optoelectronics, or even longer as it is the case of $\mathrm{Cz}$ (1.1 s) [18]. The longer lifetime compensates the slow velocity yielding a much longer diffusion length for triplet excitons reaching lengths of tens of $\mu \mathrm{m}$ as it is shown in Table III. Taking dimer $\mathrm{C}$ as an example, the diffusion constant in $\mathrm{T}_{1}$ is $D=6.810^{-6} \mathrm{~cm}^{2} \mathrm{~s}^{-1}, 4$ orders lower than in $\mathrm{S}_{1}$, and two orders lower than the reported value for anthracene $D=(0.5-2) 10^{-4} \mathrm{~cm}^{2} \mathrm{~s}^{-1}$. However, the diffusion length estimated for dimer $\mathrm{C}$ is $27 \mu \mathrm{m}, 200$ times greater than $L_{D}$ in $\mathrm{S}_{1}$ and comparable to the value measured in anthracene crystals $\left(L_{D}=7-20 \mu \mathrm{m}\right)$ [53].
This huge increase comes from the long phosphorescence lifetime of $\mathrm{T}_{1}$ for $\mathrm{Cz}$ (see Equation 8) (for anthracene $\tau_{p}$ $=23 \mathrm{~ms}$ ) [55].

The long lifetime of triplets in $\mathrm{Cz}$ enables delayed fluorescence via a triplet-triplet annihilation (TTA). Considering the $k_{i j}$ of dimer $\mathrm{C}$ and no trapping or quenching, a triplet can visit $4.510^{9}$ molecules in its lifetime period (1.1 $10^{5}$ for a singlet). However, the generation of triplets is limited by the relatively low $k_{I S C}$ which is two orders of magnitude lower than $k_{f}$ (Table I). Dopants can be used to facilitate ISC, for example, N,N-di(9H-fluoren-2-yl)phenanthrene-3-amine (DFAP) has been successfully used to increase triplettriplet annihilation delayed fluorescence (TTADF) in fluorene crystals [56].

The rate equation of the concentration of triplets $[T]$ is

$$
\frac{\partial[T]}{\partial t}=G_{T}+\left(k_{r}+k_{n r}\right)[T]+\gamma_{T T A}[T]^{2},
$$

where $G_{T}$ is the rate for the generation of triplets, $\left(k_{r}+k_{n r}\right)$ is the sum for the radiative and nonradiative decay constants of the triplet states, and $\gamma_{T T A}$ is the bimolecular annihilation constant which is related to the triplet diffusion coefficient D via Smoluchowski's theory of bimolecular reactions:

$$
\gamma_{T T A}=4 \pi R_{a} D
$$

where $R_{a}$ is the annihilation radius calculated as the average distance between two excitons undergoing annihilation.

Under the condition of a continuous generation of triplets $\left(G_{T}=\right.$ const $)$, the steady-state solution of Equation 11 produces two different regimes [53]. At low excitation power density $\left(k_{r}+k_{n r}\right)[T]>>\gamma_{T T A}[T]^{2}$ and $[T] \propto G_{T}$, the spontaneous decay governs the dynamics of excitons, whereas for high densities more triplet excitons are produced, $\gamma_{T T A}[T]^{2}>>\left(k_{r}+k_{n r}\right)[T]$ and $[T] \propto \sqrt{G_{T}}$. Especially interesting is the power density at which $\left(k_{r}+k_{n r}\right)[T]=\gamma_{T T A}[T]^{2}$ because it represents the threshold at which the TTA process starts dominating the exciton dynamics. This power density threshold can be estimated from the material photophysical constants as

$$
G_{t h} \approx \frac{1}{\gamma_{T T A} \tau^{2}},
$$

where $\tau$ is the lifetime of the excitation.

Equation 13 indicates that exciton-exciton annihilation becomes more important as the exciton lifetime is longer. Considering dimer $\mathrm{C}$, the bimolecular annihilation constant estimated for $\mathrm{Cz}$ is $\gamma_{T T A}=4.910^{-12} \mathrm{~cm}^{3}$ $\mathrm{s}^{-1}$. This value is comparable with the reported value for anthracene crystals $\gamma_{T T A}=2.1-2.410^{-11}[57]$. Despite 
the low bimolecular annihilation constant, $\mathrm{Cz}$ presents a remarkably long phosphorescence lifetime which can yield a small $G_{t h}$ to produce TTADF using relatively low powers. Delayed fluorescence has been observed for pure $\mathrm{Cz}$ crystals [20], but no systematic investigation of the effect of the temperature or the light power density has been reported so far.

\section{Isomeric impurities}

Liu et al. showed that very low concentrations of Bd (even $0.1 \mathrm{~mol} \%$ ), an impurity commonly present in commercial $\mathrm{Cz}$, originate UOP at room temperature [20]. Direct excitation and transport of singlets and triplets in the $\mathrm{Cz}$ crystal can enable the charge separation at $\mathrm{Cz} / \mathrm{Bd}$ molecular junctions. Herein, we evaluate how the presence of $\mathrm{Bd}$ isomeric defects can affect the excited state mechanism of $\mathrm{Cz}$ in the solid state.

In our calculations, we considered $\mathrm{Bd}-\mathrm{Cz}$ dimers created by substituting a $\mathrm{Cz}$ molecule by a molecule of $\mathrm{Bd}$, we reoptimised the geometries of the ground and excited states using the OEC method. Because of the alignment of the energy levels of $\mathrm{Cz}$ and $\mathrm{Bd}$, the $\mathrm{CT}$ state where $\mathrm{Cz}$ acts as an electron acceptor from $\mathrm{Bd}\left(\mathrm{Cz}^{-} \mathrm{Bd}^{+}\right)$ is more stable than the $\left(\mathrm{Cz}^{+} \mathrm{Bd}^{-}\right)$state (Tables $\mathrm{S} 9$ and S10) [20]. To analyse the role of the $\mathrm{Cz}^{-} \mathrm{Bd}^{+}$states in the electron transfer process, we optimised these states using the CDFT method for the dimers with smaller distances between the centres of mass (A-C).

Our calculations show that the presence of the $\mathrm{Bd}$ impurities redshifted the fluorescence emission energy of $\mathrm{Cz}$ around $33 \mathrm{~nm}$ (Table IV) which is in line with the $54 \mathrm{~nm}$ obtained experimentally. Both the $\mathrm{S}_{1}$ and $\mathrm{T}_{1}$ minima of the embedded $\mathrm{Cz}$-Bd dimers show a significant localisation of the excitation on the $\mathrm{Bd}$ moiety (Figures S12-S15). The calculated absorption of the Cz-Bd dimers at $3.5 \mathrm{eV}$ agrees with the onset absorption signal observed after HPLC of commercial $\mathrm{Cz}$, which has been attributed to $\mathrm{Bd}[20]$.

TABLE IV. Emission energies (in eV) for dimers A, B and C obtained with TD-B3LYP/6-311++G(d,p) within the OEC model

\begin{tabular}{|c|c|c|c|}
\hline Transition & Dimer & Dimer & Oimer \\
\hline $\mathrm{S}_{0} \rightarrow \mathrm{S}_{1}$ & 3.51 & 3.50 & 3.50 \\
\hline $\mathrm{S}_{1} \rightarrow \mathrm{S}_{0}$ & 3.14 & 3.16 & 3.16 \\
\hline $\mathrm{T}_{1} \rightarrow \mathrm{S}_{0}$ & 1.58 & 1.60 & 1.60 \\
\hline
\end{tabular}

The predicted fluorescence band of the Cz-Bd dimers appeared at 3.14-3.16 eV within the region where delayed emission of $\mathrm{Cz}$ doped with $\mathrm{Bd}$ has been observed (354-543 $\mathrm{nm})$ [20]. For dimers A and B, the energy gap between the $\mathrm{Cz}^{-} \mathrm{Bd}^{+}$state and the ground state is around 3 $\mathrm{eV}$, which is also in the same region. We explored the mechanism of formation of $\mathrm{Cz}^{-} \mathrm{Bd}^{+}$states at the $\mathrm{Cz}-$ $\mathrm{Bd}$ molecular junction using the energy profiles based on different excited state minima for dimers A-C. Herein, we focus on the analysis of dimer A (Figure 5), the energy profiles for dimers A-C can be found in Figures S12-S14.

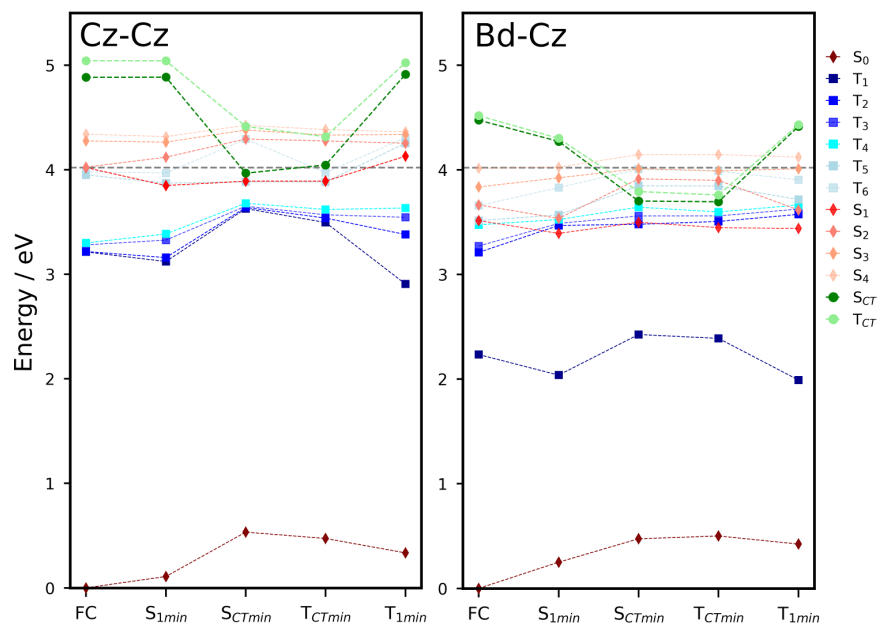

FIG. 5. Energy profiles for singlet and triplet states, including the $\mathrm{Cz}^{-} \mathrm{Cz}^{+}$and $\mathrm{Cz}^{-} \mathrm{Bd}^{+}$charge transfer states $\left(\mathrm{S}_{C T}\right.$ and $\mathrm{T}_{C T}$ ) computed at the critical points for the dimer $\mathrm{A}$ embedded in the crystal environment using the OEC model.

For pure crystalline $\mathrm{Cz}$, we have already discussed the monomer-centred mechanism in Section III A. Based on the localisation of the excitations and the energy profiles for the $\mathrm{Cz}$ dimers, we expect similar deactivation mechanisms. In this section, we analysed these decay pathways in doped dimers $(\mathrm{Cz}-\mathrm{Bd})$ contrasting them with the behaviour of pure $\mathrm{Cz}$. The mechanism for the $\mathrm{Cz}$ dimers starts with the excitation to one of the exciton states $\left(\mathrm{S}_{1}-\mathrm{S}_{4}\right)$ and analogously to the molecule-centred mechanism, vibrational relaxation is likely to stabilise the excitation in $\mathrm{S}_{1}$. For all dimers, $\mathrm{S}_{1}$ is clearly localised on one of the $\mathrm{Cz}$ monomers. In the case of $\mathrm{Cz}-\mathrm{Bd}$, for all dimers, the excitations obtained for the Franck Condon and $\mathrm{S}_{1}$ minima are localised on $\mathrm{Bd}$. Once in $\mathrm{S}_{1}$, the systems will emit light or nonradiative decay processes will be activated including exciton transport (Section III B 2) and charge transfer.

Decay to the triplet manifold through ISC is highly likely. For pure $\mathrm{Cz}$, given the proximity in energy with $\mathrm{T}_{5}$ and $\mathrm{T}_{6}$ and their similar SOCs $\left(0.36\right.$ and $0.26 \mathrm{~cm}^{-1}$ respectively) the transition could occur to any of these states (Table $\mathrm{S} 6$ ). For doped $\mathrm{Cz}$, at the $\mathrm{S}_{1}$ geometry, the $\mathrm{T}_{2}, \mathrm{~T}_{3}$ and $\mathrm{T}_{4}$ states are almost resonant with $\mathrm{S}_{1}$ making ISC very likely. For $\mathrm{B}$ and $\mathrm{C}$, the largest SOCs are obtained for $\mathrm{T}_{3}\left(0.53\right.$ and $0.45 \mathrm{~cm}^{-1}$ respectively), in the case of $\mathrm{A}$ for $\mathrm{T}_{4}\left(0.92 \mathrm{~cm}^{-1}\right)$. The SOCs are slightly larger than the obtained for pure $\mathrm{Cz}$, which suggests that the impurity could help ISC. Following the population of the triplets, internal conversion will take the system to the localised $\mathrm{T}_{1}$ state. 


\section{Charge transfer states}

From the $\mathrm{S}_{1}$ minima, another possible mechanism is the formation of charge separated states. For dimer $\mathrm{A}$, the energy of the optimised $\mathrm{Cz}^{-} \mathrm{Cz}^{+}$state in the singlet state is nearly resonant to the initial excitation energy while accessing the $\mathrm{Cz}^{-} \mathrm{Cz}^{+}$triplet requires to surpass a classical inaccessible energy barrier of around $0.4 \mathrm{eV}$. Similarly for both dimers B and C, the barriers to access the triplets are very high and classically forbidden provided initial excitation to the lower singlet manifold. While increasing the initial excitation energy or the temperature can allow populating these states, recombination is very likely. Due to the high energy of the $\mathrm{Cz}^{-} \mathrm{Cz}^{+}$state, other decay mechanisms will dominate.

How does the picture change for the $\mathrm{Cz}-\mathrm{Bd}$ molecular junctions? Both the singlet and triplet $\mathrm{Cz}^{-} \mathrm{Bd}^{+}$states are significantly more stable than in pure $\mathrm{Cz}$. For the $\mathrm{Bd}$ doped dimers $\mathrm{A}$ and $\mathrm{B}$, the energies of the $\mathrm{Cz}^{-} \mathrm{Bd}^{+}$states at their optimised geometry are below the reference excitation energy for any of the $\mathrm{S}_{1}-\mathrm{S}_{4}$ states. Small amplitude vibrational distortions allowed in the crystalline phase facilitate the access to $\mathrm{Cz}^{-} \mathrm{Bd}^{+}$states swiftly when an isomeric defect is nearby. For the dimer $\mathrm{C}, \mathrm{Cz}^{-} \mathrm{Bd}^{+}$singlets and triplets are above the initial excitation energy and will require additional energy to become accessible. Given the longer lifetime of triplet states, it is highly probable for the electron transfer to happen from the triplet state.

While the energy of the optimised geometry the $\mathrm{Cz}^{-} \mathrm{Bd}^{+}$states are slightly higher than $\mathrm{S}_{1}$, charge separation can occur because the rate of charge transfer involving $\mathrm{Cz}^{-}$for dimers $\mathrm{A}-\mathrm{D}$ is in the order of vibrational relaxation (Table $\mathrm{V}$ ). The mechanism involves the formation of $\mathrm{Cz}^{-} \mathrm{Bd}^{+}$states associated with dimers $\mathrm{A}$ or $\mathrm{B}$, followed by charge separation. The $\mathrm{Bd}^{+}$radical cations can get trapped by the defects but $\mathrm{Cz}^{-}$can transport across the crystal $\left(\mathrm{Cz}^{-}+\mathrm{Cz}=\mathrm{Cz}\right.$ $+\mathrm{Cz}^{-}$). Our calculated rates (Table V) indicate that charge separation is faster for dimer $\mathrm{C}\left(1.710^{15} \mathrm{~s}^{-1}\right.$, MLJ value), followed by dimer A $\left(4.110^{14} \mathrm{~s}^{-1}\right)$ and dimers D and B (4.1 $10^{14}$ and $\left.7.910^{13} \mathrm{~s}^{-1}\right)$. Figure 4 shows some possible pathways for electron transfer after the formation of the $\mathrm{Cz}^{-}$polarons. These processes keep the excitation alive for longer times contributing to the UOP.

The overall energy makeup indicates that the presence of isomeric impurities helps charge separation allowing for the formation of the negative charge polarons $\left(\mathrm{Cz}^{-}\right)$. In the experimental work, the absorption band at 460 $472 \mathrm{~nm}(2.6-2.7 \mathrm{eV})$ has been assigned to the $\mathrm{Cz}^{-}$ species [20]. Our calculations of the embedded $\mathrm{Cz}^{-}$have the excitation with higher oscillator strength predicted around $2.1 \mathrm{eV}$ (2.3 eV with ADC(2)/TZVP, Table S11).

For the dimer A embedded in point charges, we compared the energy levels of the $\mathrm{Cz}^{-} \mathrm{Cz}^{+}$ and $\mathrm{Cz}^{-} \mathrm{Bd}^{+}$geometries optimised with the CDFT-
TABLE V. Charge transfer $\mathrm{Cz}^{-} \rightarrow \mathrm{Cz}$ rate coefficients computed for the embedded dimers A-F using equations 2 and 3 (for the values shown in brackets).

\begin{tabular}{|c|c|}
\hline & $k_{C T}^{C z^{-}} \rightarrow c$ \\
\hline A & $7.610^{13}\left(4.110^{1}\right.$ \\
\hline & $1.410^{13}$ \\
\hline & $3.210^{14}\left(1.710^{15}\right)$ \\
\hline & $2.410^{13}\left(1.310^{14}\right)$ \\
\hline$F$ & $5.510^{12}\left(3.010^{13}\right)$ \\
\hline $\mathrm{F}$ & $6.810^{11}\left(3.710^{12}\right)$ \\
\hline
\end{tabular}

B3LYP $/ 6-311++G(d, p)$ with those calculated with ADC (2)/TZVP (Table S10). For example, the energy gaps between $\mathrm{Cz}^{-} \mathrm{Bd}^{+}$state and the ground state for the optimised geometry are 3.87 and $3.85 \mathrm{eV}$ for ADC(2)/TZVP and CDFT-B3LYP/6-311++G(d,p), respectively. Both methods confirm that both the singlets and triplets of the $\mathrm{Cz}^{-} \mathrm{Bd}^{+}$state are accessible provided initial excitation energy to $\mathrm{S}_{1}$. In the case of $\mathrm{Cz}-\mathrm{Cz}$ dimers, these states are higher in energy, which explain why charge transfer states have not been observed in pure crystalline $\mathrm{Cz}$.

\section{CONCLUSIONS}

Carbazole and its derivatives have applications in organic functional materials with long-lived excited states and ultralong phosphorescence. Due to the contradictory values of efficiencies and quantum yields obtained for materials synthesised by different groups, the interpretation of the processes underlying the UOP in $\mathrm{Cz}$ has been under debate [20]. In this paper, we explore in detail the light-activated pathways in pure crystalline carbazole and $\mathrm{Cz}$ doped with $\mathrm{Bd}$. In contrast with previously proposed mechanisms based on $\mathrm{H}$-aggregation $[19,49]$, we found that the $\mathrm{H}$ - or J-aggregation do not affect appreciably the excited state deactivation pathways or the triplet lifetimes.

The light-activated mechanism starts with the initial population of singlet states, which rapidly decay to $\mathrm{S}_{1}$ or the triplet manifold. Our estimation of fluorescence quantum yield and intersystem crossing rates are in excellent agreement with the experimental values, highlighting that ISC is the main nonradiative decay process from the singlet states. Triplet states are clearly localised on independent $\mathrm{Cz}$ molecules and their main transport mechanism is the Dexter energy transfer. Based on the estimation of hopping rates, we analysed possible singlet exciton and triplet transfer pathways. In the $\mathrm{Cz}$ crystal, exciton transport is very effective along $\mathrm{C}$ dimers, with a $\pi$-stack structure. Because of the less effective couplings between triplets and singlet states, the Dexter energy transport involving triplets is slower. However, due to the long lifetimes of triplets, their diffusion lengths are longer (at least in 2 orders of 
magnitude).

Triplets (and singlets) can travel to the Bd defects or they can be directly excited at the molecular $\mathrm{Cz}$ $\mathrm{Bd}$ junctions. The $\left(\mathrm{Cz}^{-} \mathrm{Bd}^{+}\right)$states are appreciably more stable than the $\left(\mathrm{Cz}^{-} \mathrm{Cz}^{+}\right)$states. Because of this, charge separation occurs at the $\mathrm{Cz}-\mathrm{Bd}$ molecular interfaces generating $\mathrm{Cz}^{-}$polarons. The effective charge transfer from $\mathrm{Cz}^{-}$to neutral $\mathrm{Cz}$ helps keep the excited states alive increasing the lifetimes in commercial or doped samples of crystalline $\mathrm{Cz}$.

We think our results contribute to a better understanding of light-activated mechanisms in crystalline $\mathrm{Cz}$ and the role of isomeric defects on the long-lived excited states. Controlling the functionality of organic materials by isomeric doping seems to be a promising strategy with plenty of applications in the area of optoelectronics [20]. Our simulations provide a plausible explanation for the generation of charge-separated states in line with the experimental observations. We hope this work can help support further advances in this exciting field.

\section{CONFLICTS OF INTEREST}

There are no conflicts to declare.

\section{ACKNOWLEDGEMENTS}

This research has been supported by the EPSRC (EP/R029385/1) and Leverhulme Trust (RPG-2019122). We utilized Queen Mary's Apocrita HPC facility, supported by QMUL Research-IT and the ARCHER UK National Supercomputing Service (EP/L000202/1) via the Materials Chemistry Consortium and the Molecular Modelling Hub for computational resources, MMM Hub, which is partially funded by EPSRC (EP/T022213/1). The authors acknowledge the support from the School of Biological and Chemical Sciences at the Queen Mary University of London.

\section{SUPPORTING INFORMATION AVAILABLE}

[1] J. Mei, N. L. Leung, R. T. Kwok, J. W. Lam, and B. Z. Tang, Chemical Reviews 115, 11718 (2015).

[2] S. Hirata, Advanced Optical Materials 5, 1700116 (2017).

[3] Y. Chen, J. W. Lam, R. T. Kwok, B. Liu, and B. Z. Tang, Materials Horizons 6, 428 (2019).

[4] R. Crespo-Otero, Q. Li, and L. Blancafort, Chemistry An Asian Journal 14, 700 (2019).

[5] J. Li and K. Pu, Chemical Society Reviews 48, 38 (2019).

[6] I. Bergenti, V. Dediu, M. Prezioso, and A. Riminucci, Philosophical Transactions of the Royal Society A: Mathematical, Physical and Engineering Sciences 369, 3054 (2011).

[7] J. Gierschner, S. Varghese, and S. Y. Park, Advanced Optical Materials 4, 348 (2016).

[8] W. Jia, Q. Wang, H. Shi, Z. An, and W. Huang, Chemistry - A European Journal 26, 4437 (2020).

[9] Y. Gong, L. Zhao, Q. Peng, D. Fan, W. Z. Yuan, Y. Zhang, and B. Z. Tang, Chemical Science 6, 4438 (2015).

[10] T. Zhang, H. Gao, A. Lv, Z. Wang, Y. Gong, D. Ding, H. Ma, Y. Zhang, and W. Z. Yuan, Journal of Materials Chemistry C 7, 9095 (2019).

[11] X. Wang, H. Ma, M. Gu, C. Lin, N. Gan, Z. Xie, H. Wang, L. Bian, L. Fu, S. Cai, Z. Chi, W. Yao, Z. An, H. Shi, and W. Huang, Chemistry of Materials 31, 5584 (2019).

[12] K. Jiang, L. Zhang, J. Lu, C. Xu, C. Cai, and H. Lin, Angewandte Chemie - International Edition 55, 7231 (2016).

[13] Y. Su, Y. Zhang, Z. Wang, W. Gao, P. Jia, D. Zhang, C. Yang, Y. Li, and Y. Zhao, Angewandte Chemie International Edition 59, 9967 (2020).
[14] X. Zhen, Y. Tao, Z. An, P. Chen, C. Xu, R. Chen, W. Huang, and K. Pu, Advanced Materials 29, 1606665 (2017).

[15] Kenry, C. Chen, and B. Liu, Nature Communications 10, 1 (2019).

[16] H. A. Collins, M. Khurana, E. H. Moriyama, A. Mariampillai, E. Dahlstedt, M. Balaz, M. K. Kuimova, M. Drobizhev, V. X. Yang, D. Phillips, A. Rebane, B. C. Wilson, and H. L. Anderson, Nature Photonics 2, 420 (2008).

[17] R. Kabe, N. Notsuka, K. Yoshida, and C. Adachi, Advanced Materials 28, 655 (2016).

[18] W. Zhao, T. S. Cheung, N. Jiang, W. Huang, J. W. Lam, X. Zhang, Z. He, and B. Z. Tang, Nature Communications 10, 1595 (2019).

[19] C. Sun, X. Ran, X. Wang, Z. Cheng, Q. Wu, S. Cai, L. Gu, N. Gan, H. Shi, Z. An, H. Shi, and W. Huang, Journal of Physical Chemistry Letters 9, 335 (2018).

[20] C. Chen, Z. Chi, K. C. Chong, A. S. Batsanov, Z. Yang, Z. Mao, Z. Yang, and B. Liu, Nature Materials, 4 (2020).

[21] A. Troisi, Chemical Society Reviews 40, 2347 (2011).

[22] P. Giannozzi, S. Baroni, N. Bonini, M. Calandra, R. Car, C. Cavazzoni, D. Ceresoli, G. L. Chiarotti, M. Cococcioni, I. Dabo, A. Dal Corso, S. De Gironcoli, S. Fabris, G. Fratesi, R. Gebauer, U. Gerstmann, C. Gougoussis, A. Kokalj, M. Lazzeri, L. MartinSamos, N. Marzari, F. Mauri, R. Mazzarello, S. Paolini, A. Pasquarello, L. Paulatto, C. Sbraccia, S. Scandolo, G. Sclauzero, A. P. Seitsonen, A. Smogunov, P. Umari, and R. M. Wentzcovitch, Journal of Physics Condensed Matter 21, 395502 (2009). 
[23] M. Rivera, M. Dommett, and R. Crespo-Otero, Journal of Chemical Theory and Computation 15, 2504 (2019).

[24] M. Rivera, M. Dommett, A. Sidat, W. Rahim, and R. Crespo-Otero, Journal of Computational Chemistry 41, 1045 (2020).

[25] M. J. Frisch, G. W. Trucks, H. B. Schlegel, G. E. Scuseria, M. A. Robb, J. R. Cheeseman, G. Scalmani, V. Barone, G. A. Petersson, H. Nakatsuji, X. Li, M. Caricato, A. V. Marenich, J. Bloino, B. G. Janesko, R. Gomperts, B. Mennucci, H. P. Hratchian, J. V. Ortiz, A. F. Izmaylov, J. L. Sonnenberg, D. WilliamsYoung, F. Ding, F. Lipparini, F. Egidi, J. Goings, B. Peng, A. Petrone, T. Henderson, D. Ranasinghe, V. G. Zakrzewski, J. Gao, N. Rega, G. Zheng, W. Liang, M. Hada, M. Ehara, K. Toyota, R. Fukuda, J. Hasegawa, M. Ishida, T. Nakajima, Y. Honda, O. Kitao, H. Nakai, T. Vreven, K. Throssell, J. A. Montgomery, Jr., J. E. Peralta, F. Ogliaro, M. J. Bearpark, J. J. Heyd, E. N. Brothers, K. N. Kudin, V. N. Staroverov, T. A. Keith, R. Kobayashi, J. Normand, K. Raghavachari, A. P. Rendell, J. C. Burant, S. S. Iyengar, J. Tomasi, M. Cossi, J. M. Millam, M. Klene, C. Adamo, R. Cammi, J. W. Ochterski, R. L. Martin, K. Morokuma, O. Farkas, J. B. Foresman, and D. J. Fox, Gaussian ${ }^{16}$ Revision A.03 (2016), gaussian Inc. Wallingford CT.

[26] B. Aradi, B. Hourahine, and T. Frauenheim, Journal of Physical Chemistry A 111, 5678 (2007).

[27] B. G. Levine, J. D. Coe, and T. J. Martínez, The Journal of Physical Chemistry B 112, 405 (2008).

[28] G. C. Schatz and M. A. Ratner, Quantum Mechanics in Chemistry, 09 (1998).

[29] W. J. Kusto and J. W. Rohleder, Molecular crystals and liquid crystals 55, 151 (1979).

[30] G. Baryshnikov, B. Minaev, and H. Ågren, Chemical Reviews 117, 6500 (2017).

[31] H. Ågren, O. Vahtras, and B. Minaev, Advances in Quantum Chemistry 27, 71 (1996).

[32] B. Minaev, G. Baryshnikov, and H. Agren, Physical Chemistry Chemical Physics 16, 1719 (2014).

[33] O. Vahtras, H. Ågren, P. Jørgensen, H. J. A. Jensen, T. Helgaker, and J. Olsen, The Journal of Chemical Physics 97, 9178 (1992).

[34] K. Aidas, C. Angeli, K. L. Bak, V. Bakken, R. Bast, L. Boman, O. Christiansen, R. Cimiraglia, S. Coriani, P. Dahle, E. K. Dalskov, U. Ekström, T. Enevoldsen, J. J. Eriksen, P. Ettenhuber, B. Fernández, L. Ferrighi, H. Fliegl, L. Frediani, K. Hald, A. Halkier, C. Hättig, H. Heiberg, T. Helgaker, A. C. Hennum, H. Hettema, E. Hjertenæs, S. Høst, I.-M. Høyvik, M. F. Iozzi, B. Jansík, H. J. Aa. Jensen, D. Jonsson, P. Jørgensen, J. Kauczor, S. Kirpekar, T. Kjærgaard, W. Klopper, S. Knecht, R. Kobayashi, H. Koch, J. Kongsted, A. Krapp, K. Kristensen, A. Ligabue, O. B. Lutnæs, J. I. Melo, K. V. Mikkelsen, R. H. Myhre, C. Neiss, C. B. Nielsen, P. Norman, J. Olsen, J. M. H. Olsen, A. Osted, M. J. Packer, F. Pawlowski, T. B. Pedersen, P. F. Provasi, S. Reine, Z. Rinkevicius, T. A. Ruden, K. Ruud, V. V. Rybkin, P. Sałek, C. C. M. Samson, A. S. de Merás, T. Saue, S. P. A. Sauer, B. Schimmelpfennig, K. Sneskov, A. H. Steindal, K. O. Sylvester-Hvid, P. R. Taylor, A. M. Teale, E. I. Tellgren, D. P. Tew, A. J. Thorvaldsen, L. Thøgersen, O. Vahtras, M. A. Watson, D. J. D. Wilson, M. Ziolkowski, and H. Ågren, WIREs Comput. Mol. Sci. 4, 269 (2014).
[35] R. Liu, X. Gao, M. Barbatti, J. Jiang, and G. Zhang, Journal of Physical Chemistry Letters 10, 1388 (2019).

[36] X. Gao, S. Bai, D. Fazzi, T. Niehaus, M. Barbatti, and W. Thiel, Journal of Chemical Theory and Computation 13, 515 (2017).

[37] R. P. Fornari, J. Aragó, and A. Troisi, The Journal of Chemical Physics 142, 184105 (2015).

[38] J. Aragó and A. Troisi, Advanced Functional Materials 26, 2316 (2016).

[39] J. R. Reimers, Journal of Chemical Physics 115, 9103 (2001).

[40] Y. Shao, Z. Gan, E. Epifanovsky, A. T. Gilbert, M. Wormit, J. Kussmann, A. W. Lange, A. Behn, J. Deng, X. Feng, D. Ghosh, M. Goldey, P. R. Horn, L. D. Jacobson, I. Kaliman, R. Z. Khaliullin, T. Kuś, A. Landau, J. Liu, E. I. Proynov, Y. M. Rhee, R. M. Richard, M. A. Rohrdanz, R. P. Steele, E. J. Sundstrom, H. L. Woodcock, P. M. Zimmerman, D. Zuev, B. Albrecht, E. Alguire, B. Austin, G. J. O. Beran, Y. A. Bernard, E. Berquist, K. Brandhorst, K. B. Bravaya, S. T. Brown, D. Casanova, C.-M. Chang, Y. Chen, S. H. Chien, K. D. Closser, D. L. Crittenden, M. Diedenhofen, R. A. DiStasio, H. Do, A. D. Dutoi, R. G. Edgar, S. Fatehi, L. Fusti-Molnar, A. Ghysels, A. Golubeva-Zadorozhnaya, J. Gomes, M. W. Hanson-Heine, P. H. Harbach, A. W. Hauser, E. G. Hohenstein, Z. C. Holden, T.-C. Jagau, H. Ji, B. Kaduk, K. Khistyaev, J. Kim, J. Kim, R. A. King, P. Klunzinger, D. Kosenkov, T. Kowalczyk, C. M. Krauter, K. U. Lao, A. D. Laurent, K. V. Lawler, S. V. Levchenko, C. Y. Lin, F. Liu, E. Livshits, R. C. Lochan, A. Luenser, P. Manohar, S. F. Manzer, S.-P. Mao, N. Mardirossian, A. V. Marenich, S. A. Maurer, N. J. Mayhall, E. Neuscamman, C. M. Oana, R. Olivares-Amaya, D. P. O'Neill, J. A. Parkhill, T. M. Perrine, R. Peverati, A. Prociuk, D. R. Rehn, E. Rosta, N. J. Russ, S. M. Sharada, S. Sharma, D. W. Small, A. Sodt, T. Stein, D. Stück, Y.-C. Su, A. J. Thom, T. Tsuchimochi, V. Vanovschi, L. Vogt, O. Vydrov, T. Wang, M. A. Watson, J. Wenzel, A. White, C. F. Williams, J. Yang, S. Yeganeh, S. R. Yost, Z.-Q. You, I. Y. Zhang, X. Zhang, Y. Zhao, B. R. Brooks, G. K. Chan, D. M. Chipman, C. J. Cramer, W. A. Goddard, M. S. Gordon, W. J. Hehre, A. Klamt, H. F. Schaefer, M. W. Schmidt, C. D. Sherrill, D. G. Truhlar, A. Warshel, X. Xu, A. Aspuru-Guzik, R. Baer, A. T. Bell, N. A. Besley, J.-D. Chai, A. Dreuw, B. D. Dunietz, T. R. Furlani, S. R. Gwaltney, C.-P. Hsu, Y. Jung, J. Kong, D. S. Lambrecht, W. Liang, C. Ochsenfeld, V. A. Rassolov, L. V. Slipchenko, J. E. Subotnik, T. Van Voorhis, J. M. Herbert, A. I. Krylov, P. M. Gill, and M. Head-Gordon, Molecular Physics 113, 184 (2015).

[41] TURBOMOLE V7.0 2015, a development of University of Karlsruhe and Forschungszentrum Karlsruhe $\mathrm{GmbH}$, 1989-2007, TURBOMOLE GmbH, since 2007; available from http://www.turbomole.com.

[42] A. R. Auty, A. C. Jones, and D. Phillips, Chemical Physics 103, 163 (1986).

[43] S. M. Bonesi and R. Erra-Balsells, Journal of Luminescence 93, 51 (2001).

[44] R. C. Powell and Z. G. Soos, Journal of Luminescence 11, 1 (1975).

[45] V. Stehr, R. F. Fink, B. Engels, J. Pflaum, and C. Deibel, Journal of Chemical Theory and Computation 10, 1242 
(2014)

[46] Y. Gu, K. Wang, Y. Dai, G. Xiao, Y. Ma, Y. Qiao, and B. Zou, Journal of Physical Chemistry Letters 8, 4191 (2017).

[47] H. J. Haink and J. R. Huber, Journal of Molecular Spectroscopy 60, 31 (1976).

[48] Z. He, W. Zhao, J. W. Lam, Q. Peng, H. Ma, G. Liang, Z. Shuai, and B. Z. Tang, Nature Communications 8, 1 (2017).

[49] Z. An, C. Zheng, Y. Tao, R. Chen, H. Shi, T. Chen, Z. Wang, H. Li, R. Deng, X. Liu, and W. Huang, Nature Materials 14, 685 (2015).

[50] M. Kasha, H. R. Rawls, and M. A. El-Bayoumi, Pure and Applied Chemistry 11, 371 (1965).
[51] N. J. Hestand and F. C. Spano, Chemical Reviews 118, 7069 (2018).

[52] H. Haink and J. Huber, Journal of Molecular Spectroscopy 60, 31 (1976).

[53] O. V. Mikhnenko, P. W. M. Blom, and T.-Q. Nguyen, Energy Environ. Sci. 8, 1867 (2015).

[54] S. R. Yost, E. Hontz, S. Yeganeh, and T. Van Voorhis, Journal of Physical Chemistry C 116, 17369 (2012).

[55] V. Ern, Phys. Rev. Lett. 22, 343 (1969).

[56] S. Hirata, H. Hara, and I. Bhattacharjee, The Journal of Physical Chemistry C 124, 25121 (2020).

[57] A. Monguzzi, J. Mezyk, F. Scotognella, R. Tubino, and F. Meinardi, Phys. Rev. B 78, 195112 (2008). 